\title{
Accuracy of a Portable Multisensor Body Monitor for Predicting Resting Energy Expenditure in Older People: A Comparison with Indirect Calorimetry
}

\author{
Stefanie Heiermann ${ }^{a}$ Kerstin Khalaj Hedayati ${ }^{a}$ Manfred J. Müller ${ }^{b}$ \\ Manuela Dittmar ${ }^{\mathrm{a}}$

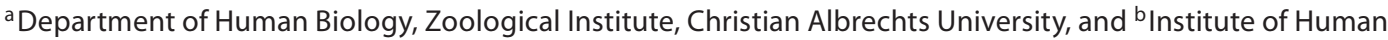 \\ Nutrition and Food Sciences, Christian Albrechts University, Kiel, Germany
}

\section{Key Words}

Accelerometry $\cdot$ Motion sensor $\cdot$ Validity $\cdot$ Indirect

calorimetry $\cdot$ Resting energy expenditure

\begin{abstract}
Background: Accurate and comfortable methods are needed to determine resting energy expenditure (REE) in older people who are characterized by a lowered metabolic rate. The portable SenseWear ${ }^{\circledR}$ armband (SWA) body monitor, worn on the right upper arm, can easily be used by this age group in an ambulatory manner. Objective: The purpose of this study was to evaluate the reliability and accuracy of the SWA armband in determining REE in healthy, normal-weight older people. Methods: Participants were 49 older, community-dwelling volunteers aged 60-87 years. Reliability was tested in all subjects. Accuracy was analyzed in 32 subjects by comparing REE estimated by SWA against REE measured by indirect calorimetry as a criterion method. Data were simultaneously and continuously recorded for $20 \mathrm{~min}$ in the morning. In the same subjects, REE determined by SWA from night recording was evaluated for accuracy against indirect calorimetry. The agreement between methods was assessed by the Bland-Altman procedure. Results: (1) REE measured by SWA was reliable when comparing 2 consecutive measurements (typical error: 1.9\%). (2) REE calculated from morn-
\end{abstract}

ing recording $(1,543 \pm 181 \mathrm{kcal} / 24 \mathrm{~h})$ was higher than REE from night recording $(1,564 \pm 192 \mathrm{kcal} / 24 \mathrm{~h} ; \mathrm{p}=0.018)$. (3) Compared with REE by indirect calorimetry (1,377 \pm 228 $\mathrm{kcal} / 24 \mathrm{~h}$ ) for accuracy, the SWA overestimated mean REE in the older people by 12 and $14 \%$, respectively, for morning and night recording (each $p<0.001)$. Conclusions: The SWA provides a reliable estimate of REE in healthy older subjects and has the advantage of easy handling. The 20-min recording time, which was recommended by the manufacturer, can be applied. However, the SWA overestimates REE in this group, possibly due to age-related changes in skin conductance and thermoregulation, both being measured by sensors of the SWA armband. This requires improving the SWA by developing better fitting algorithms for predicting REE in older people.

Copyright $\odot 2011$ S. Karger AG, Basel

\section{Introduction}

Resting energy expenditure (REE) is reduced in older people due to disproportionate age-related loss of metabolically active organ tissue mass and increase in fat mass [1]. It amounts to $65-70 \%$ of total energy expenditure [2]. The decline in REE is considered as a risk factor for frailty and morbidity in the elderly [3]. The accurate determi-

\section{KARGER \\ Fax +4161306 1234 \\ E-Mail karger@karger.ch}

www.karger.com
(C) 2011 S. Karger AG, Basel

0304-324X/11/0575-0473\$38.00/0

Accessible online at:

www.karger.com/ger
Prof. Dr. Manuela Dittmar

Department of Human Biology, Zoological Institute, Christian Albrechts University Am Botanischen Garten 9

DE-24118 Kiel (Germany)

Tel. +49431 880 4357, Fax +49 431880 2795, E-Mail mdittmar@zoologie.uni-kiel.de 
nation of REE is a critical component for weight management and, thereby, for the prevention of lifestyle-related health problems in older people. The gold standard method for determining REE is indirect calorimetry. However, this method is costly and cumbersome, and measurements are primarily restricted to research laboratories. For older people, ambulatory measurements are more comfortable. There is a need for an objective and comfortable device to determine REE in older persons, usable at home during daily activities. The SenseWear ${ }^{\circledR}$ armband (SWA) seems well suited because this wireless, small-sized body monitor is worn on the upper arm and can be used in the free-living environment with easy handling. The only effort it requires by the user is to be attached and to be worn throughout day and night. In contrast to available accelerometers, it gathers data not only on body motion but also on various physiological parameters, in particular on heat production of the body as a result of REE, due to additional heat flux and temperature sensors which are incorporated into the device. However, validation studies on REE determination by SWA in older adults are completely missing. Existing validation studies are restricted to young and middle-aged healthy adults $[4,5]$, obese individuals [6] or patients in various disease states $[7,8]$. We suppose that the SWA device may lead to different results in older populations due to age-related changes in their skin and body composition [9]. With age, the fat-free mass decreases, resulting in a loss of metabolically active components. Also, the skin of older persons becomes thinner and dryer, sweating is reduced, and vascular supply declines [10]. This affects skin conductance and thermoregulation, both measured by sensors of the SWA. Based on these age-related changes, we assume that the SWA algorithms developed for younger adults cannot be applied to older ones.

Therefore, the primary objective of this study was to validate the SWA for assessing REE against indirect calorimetry as a criterion method in a sample of healthy, community-dwelling older people. A second objective was to compare REE estimated by SWA from different recording times (morning vs. night). The advantage of determining REE from night recording is that it is more comfortable for older subjects than morning recording. For night recording, the subjects do not have to come to the laboratory and meet pretest conditions such as overnight fasting, as is the case with morning recording. This is important for field studies because many older people do not agree to come to the university laboratory after an overnight fast to measure REE.

\section{Subjects and Methods}

\section{Subjects}

A total of 49 healthy, community-dwelling volunteers (24 males and 25 females) aged 60-87 years (BMI males: $27.3 \pm 2.53$; females: $27.0 \pm 4.25$ ) were recruited by university lectures for older people, announcements in local newspapers, and flyers distributed in Kiel, Germany, to participate in the study. Exclusion criteria were any conditions requiring medications that would affect REE, e.g. presence of thyroid diseases, asthma or chronic obstructive pulmonary disease, as assessed from the medical history. Except for 1 participant, all subjects were nonsmokers. Reliability was tested in all 49 subjects. Thirty-two volunteers (13 males and 19 females) aged 61-83 years agreed to participate in the validation part of the study. The study protocol was approved by the local ethics committee at the University of Kiel. The protocol and aim of the study were fully explained to the volunteers, who gave their written informed consent before data collection.

\section{Methods}

REE was measured by SWA and indirect calorimetry with simultaneous data recording in the morning for $20 \mathrm{~min}$ by trained persons. The volunteers arrived at the laboratory in the morning, after an overnight fast of at least $12 \mathrm{~h}$, and after having abstained from alcohol consumption and physical activity for $24 \mathrm{~h}$. REE was additionally determined from night recording by SWA.

\section{SWA Body Monitor}

REE was measured with the SenseWear $\mathrm{Pro}_{2}$ Armband body monitor (BodyMedia Inc., Pittsburgh, Pa., USA). This device is placed on the lateral head of the triceps of the right upper arm, allowing continuous body monitoring outside the laboratory with respect to the energy expenditure of a person. The SWA device determines REE by a biaxial accelerometer and various temperature sensors. In the current study, data were recorded for $24 \mathrm{~h}$ (range: 23-27 h). Recorded data were downloaded and analyzed by the associated research software InnerView Professional version 5.0. REE is calculated from the recorded physiological signals in the SWA and from demographic characteristics of the subject (age, sex, weight and stature) by using a proprietary algorithm. We assessed REE from morning recording and from night recording. With respect to morning recording, REE was assessed from a 20 min measurement, performed simultaneously with indirect calorimetric measurement, when the subject was awake and recumbent. The REE was predicted by extrapolating the energy expenditure during the 20 -min recording time to $24 \mathrm{~h}$. In addition, REE was estimated from night recording of the sleeping subject. The energy expenditure during $1 \mathrm{~h}$ of early sleep was extrapolated to $24 \mathrm{~h}$ according to the instructions of the manufacturer. For both morning and night recordings, the REE was predicted from the physiological parameters.

Indirect calorimetry was taken as a criterion method using the Vmax Spectra indirect calorimeter (SensorMedics Viasys Healthcare, Bilthoven, The Netherlands; Vmax software version 12-1A). Detailed descriptions of the device and the measurement procedure are provided elsewhere [11]. In brief, this ventilated hood system is connected with a ventilator interface and a gas-analyzing system. The flowmeter and gas analyzers are calibrated prior to each measurement. During measurement, the gas analyzers are autocalibrated every $5 \mathrm{~min}$. After resting for $10 \mathrm{~min}$, data were 
recorded from the participants at constant room temperature $\left(22^{\circ} \mathrm{C}\right)$ and humidity $(55 \%)$ for at least $30 \mathrm{~min}$ between 8.15 and 10.40 a.m. The subjects wore indoor clothes without shoes or jackets. During measurement, the subjects lay under the ventilated hood and the airflow was approximately 30 liters/min in order to keep the $\mathrm{CO}_{2}$ concentration in the expired air within a range of $0.6-0.9 \%$. Airflow, fractions of $\mathrm{O}_{2}$ (in percent) and $\mathrm{CO}_{2}$ (in percent) in the inspired and expired air, $\mathrm{V} \mathrm{O}_{2}$ (in liters per minute), $\dot{\mathrm{V}} \mathrm{O}_{2} / \mathrm{kg}$ (in milliliters per kilogram per minute), $\dot{\mathrm{V}} \mathrm{CO}_{2}$ (in liters per minute) and the respiratory quotient (RQ) were recorded every $20 \mathrm{~s}$. The initial $10 \mathrm{~min}$ of expired gas data were discarded, and only the final 20 min were used for determining REE. Values with an RQ $>1.0$ were excluded for possible hyperventilation. The data were analyzed considering steady-state criteria for $\dot{\mathrm{V}} \mathrm{O}_{2}$ and $\dot{\mathrm{V}} \mathrm{CO}_{2}$ variation of $\leq 10 \% / \mathrm{min}$ [12]. REE (in kilocalories per $24 \mathrm{~h}$ ) was computed from $\dot{\mathrm{V}} \mathrm{O}_{2}$ and $\dot{\mathrm{V}} \mathrm{CO}_{2}$ by using the abbreviated equation by Weir $[13,14]$ as follows: REE $(\mathrm{kcal} / 24 \mathrm{~h})=\left[3.94 \times \dot{\mathrm{V}} \mathrm{O}_{2}(\mathrm{ml} /\right.$ $\mathrm{min})+1.1 \times \dot{\mathrm{V} \mathrm{CO}}(\mathrm{ml} / \mathrm{min})] \times 1.44$. The REE measurements with the Vmax Spectra are reliable, showing a coefficient of variation of 5.0-5.6\% [15]. The measurements are also accurate with regard to the specifications given by Compher et al. [16].

\section{Anthropometry and Body Composition}

Stature was measured to the nearest $0.1 \mathrm{~cm}$, using an anthropometer. Body weight was determined to the nearest $0.1 \mathrm{~kg}$ with an electronic platform scale (TBF-538; Tanita Corp., Tokyo, Japan). The BMI was calculated as weight in kilograms per height in meters squared. Fat-free mass and fat mass were estimated by using a tetrapolar whole-body bioelectrical impedance analyzer (Nutriguard-M; Data Input Company, Darmstadt, Germany) and Nutri-Plus 5.1 software [17].

\section{Lifestyle Parameters}

The physical activity level of the older subjects was determined by a standardized questionnaire [18] which was developed to measure habitual physical activity in the elderly. It considers household, sport and leisure time activities over a period of 1 year, resulting in a global activity score. All items result in a separate score that incorporates activity duration, frequency and an intensity code based on energy costs. Summing the household score, sport score and leisure time activity score results in a unitless continuous global activity score.

\section{Statistical Analysis}

Statistical analyses were conducted using the software package SPSS version 15 (SPSS Inc., Chicago, Ill., USA) and MedCalc software version 7.2.0.2 (MedCalc Software, Mariakerke, Belgium). The REE by indirect calorimetry was established as the criterion measure. The data from the SWA were analyzed using the longitudinal and transverse axes counts, as well as estimates of total energy expenditure which were derived from associated software and proprietary formulas. Normality of data distribution was tested by the Kolmogorov-Smirnov test. Data are given as means \pm standard deviations (SD). Reliability was tested by determining the typical error from duplicate measurements. It is calculated as the SD of the difference scores of repeated measurements, divided by the square root of 2 . The typical error was expressed as an absolute value and as a percentage. Differences between REE values by SWA and indirect calorimetry were analyzed using paired sample t tests. Bland-Altman plots [19] were
Table 1. Descriptive physical characteristics of the older participants

\begin{tabular}{lcc}
\hline & Males $(\mathrm{n}=13)$ & Females $(\mathrm{n}=19)$ \\
\hline Age, years & $67.9 \pm 5.11$ & $69.2 \pm 5.10$ \\
Stature, cm & $175.6 \pm 3.19$ & $161.7 \pm 7.22$ \\
Weight, kg & $81.6 \pm 8.63$ & $69.7 \pm 15.03$ \\
BMI & $26.4 \pm 2.57$ & $26.4 \pm 3.74$ \\
FFM, kg & $63.5 \pm 4.99$ & $45.0 \pm 5.35$ \\
FM, kg & $18.1 \pm 5.11$ & $24.7 \pm 10.09$ \\
Physical activity level, score & $16.0 \pm 5.06$ & $14.8 \pm 7.68$ \\
\hline
\end{tabular}

Values denote means \pm SD. FFM = Fat-free mass; FM = fat mass.

created for method comparison at an individual level, determining the agreement between SWA and indirect calorimetry in predicting REE by calculating bias and limits of agreement. The latter were confidence intervals which were established as $1.96 \mathrm{SD}$ from mean difference. Pearson's product-moment correlation coefficients were calculated to analyze the relationship between method difference and various variables. Statistical significance was considered at a two-sided $\mathrm{p}<0.05$.

\section{Results}

Descriptive physical characteristics of the older participants are provided in table 1 . The BMI of most females (63.2\%) and males (61.5\%) was within the range of normal older persons (18.5-27.5), following the criteria by Müller et al. [20]. Their physical activity level was in an upper midrange for this age group [18].

\section{Reliability of SWA Measurements for Estimating REE}

The reliability of REE measurement by SWA was determined in 49 subjects from 2 consecutive night recordings. The mean values of repeated measures (first measure: $1,620.7 \pm 231.9 \mathrm{kcal} / 24 \mathrm{~h}$; second measure: 1,626.8 $\pm 228 \mathrm{kcal} / 24 \mathrm{~h})$ were not significantly different $(\mathrm{t}=$ $-0.97 ; \mathrm{p}=0.335)$, and the correlation between both measures was highly significant $(\mathrm{r}=0.98, \mathrm{p}<0.001)$. The typical error was only $30.8 \mathrm{kcal} / 24 \mathrm{~h}$ (1.9\%), indicating that the measurement of REE by SWA is reliable.

\section{Comparison of Different SWA Measurement \\ Procedures for Estimating REE}

For the SWA, morning and night recordings were compared for determining REE in the older subjects. The REE estimated from morning and from night recordings 

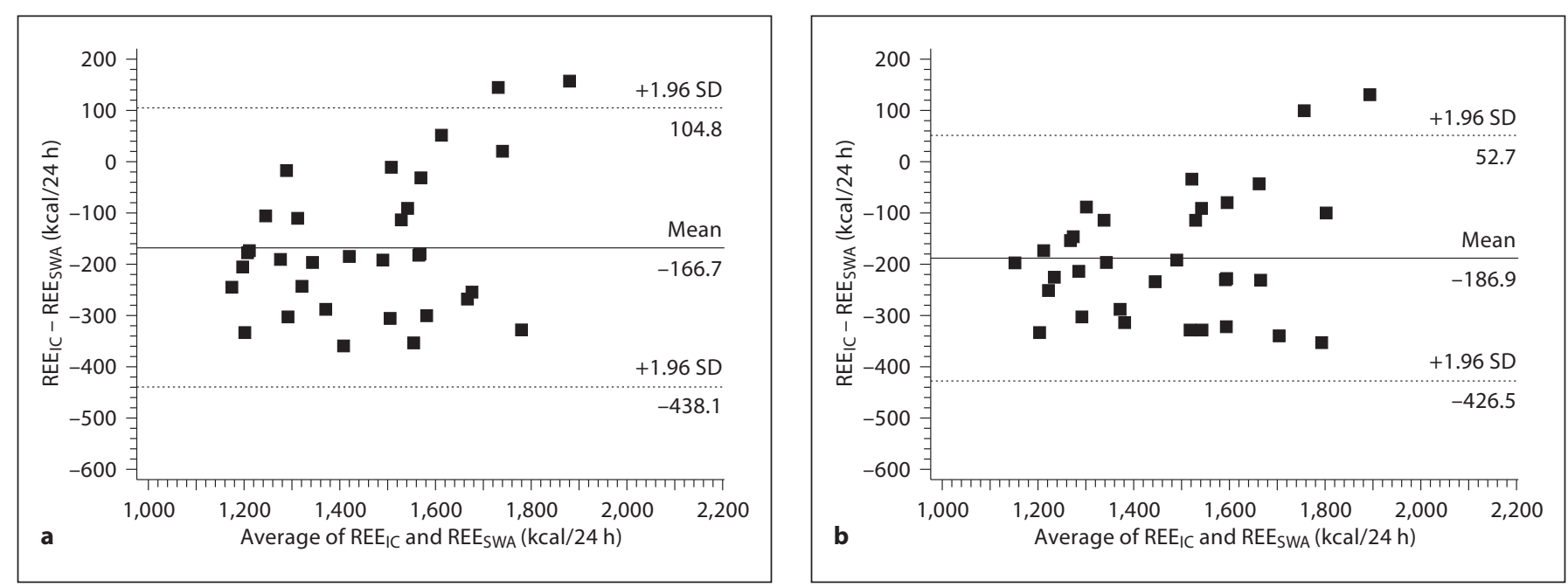

Fig. 1. Differences between REE by indirect calorimetry and REE by SWA in 32 healthy older subjects, as shown by Bland-Altman plots. The average of 2 estimates of REE (x-axis) is plotted against the difference between the 2 estimates (y-axis). For the SWA, data are given for morning (a) and night recordings (b). Solid horizontal line: mean difference between methods. Broken lines: 95\% limits of agreement. $\mathrm{REE}_{\mathrm{IC}}=\mathrm{REE}$ assessed by indirect calorimetry; $\mathrm{REE}_{\mathrm{SWA}}=\mathrm{REE}$ assessed by SWA.

Table 2. Comparison of REE measurement by SWA versus REE measurement by indirect calorimetry in 32 healthy older people

\begin{tabular}{|c|c|c|c|c|c|c|}
\hline & \multicolumn{2}{|l|}{ REE } & \multicolumn{4}{|c|}{ Method comparison } \\
\hline $\begin{array}{l}\text { Indirect calorimetry } \\
\text { SWA }\end{array}$ & $1,376.8 \pm 228.2$ & - & - & - & - & - \\
\hline REE calculated from morning recording (20 min) & $1,543.5 \pm 181.0$ & 12.1 & -6.81 & $<0.001$ & $167 \pm 271$ & -438 to 105 \\
\hline
\end{tabular}

${ }^{\mathrm{a}}$ Mean $\pm \mathrm{SD} .{ }^{\mathrm{b}}$ Mean difference in REE between SWA and indirect calorimetry. ${ }^{\mathrm{c}}$ Paired samples $\mathrm{t}$ test. ${ }^{\mathrm{d}}$ Mean difference in $\mathrm{REE} \pm$ bias between SWA and indirect calorimetry. Bias is $\pm 1.96 \mathrm{SD}$ of method difference. ${ }^{\mathrm{e}}$ Limits of agreement are lower and upper $1.96 \mathrm{SD}$.

was $1,543.5 \pm 181.0 \mathrm{kcal} / 24 \mathrm{~h}$ and $1,563.8 \pm 192.10$ $\mathrm{kcal} / 24 \mathrm{~h}$, respectively. The REE from morning recordings differed from that based on night recordings $(t=$ $-2.49 ; \mathrm{p}=0.018$ ), with higher REE values from night recordings.

\section{Accuracy of SWA Measurements for Estimating REE}

Based on the above findings, REE assessments from morning and night recording, using physiological signals, were compared with indirect calorimetry for accuracy. Table 2 shows that the SWA device significantly overpredicted the mean REE compared with indirect calorimetry. The mean overpredictions for morning and night recordings were 12 and $14 \%$, respectively. The method difference in REE correlated positively and significantly with REE by indirect calorimetry (REE from morning recording: $\mathrm{r}=0.61, \mathrm{p}<0.001$; REE from night recording: $\mathrm{r}=0.54, \mathrm{p}=0.001$ ). By contrast, the method difference did not significantly correlate with age, BMI, fat-free mass or physical activity level of the subjects (each $p>0.05)$. Figure 1 displays the overprediction of REE by SWA at an individual level. Bland-Altman plots revealed 
a greater spread of values for predictions of REE from morning recording than from night recording. An individual error greater than $\pm 20 \%$ was observed for REE morning recording and REE night recording in 28 and $31 \%$ of the subjects, respectively. This means that the individual error was somewhat greater for REE predictions from night recording.

\section{Discussion}

This study is the first one to evaluate the reliability and accuracy of the SWA device in estimating REE in healthy older people, compared with indirect calorimetry.

\section{Reliability of REE Measurement by SWA}

The results demonstrated a good reproducibility of REE by the SWA device, showing only a small typical error of $1.9 \%$. The correlation coefficient between the repeated measurements, obtained by SWA in the older healthy subjects $(r=0.98 ; \mathrm{p}<0.001)$, was comparable to values in younger healthy adults $(\mathrm{r}=0.86 ; \mathrm{p}<0.001)[5]$ and obese subjects $(r=0.88 ; \mathrm{p}<0.001)[6]$.

\section{Comparison of Morning and Night Recordings for Estimating REE by SWA}

Night recording yielded higher estimates of REE than morning recording. This could be explained by several mechanisms. First, the normal sleeping metabolic rate may be higher than the basal metabolic rate because of several factors which could modulate the sleeping metabolic rate [21]. Energy expenditure differs among the different sleep stages, being greater during REM sleep than during sleep stages 2 and 3/4 [22], probably because of increased cerebral blood flow, glucose uptake and oxygen during REM sleep. However, sleep stages have been reported to have only a small effect on the variability in metabolic rates while asleep [23]. Nevertheless, it could be crucial for calculating REE by extrapolating 1 hour of measuring to $24 \mathrm{~h}$, depending on the actual sleep stage. Second, the sleep of older people more often exhibits interruptions, brief awakenings and toilet visits than sleep of children or younger adults [24], and deep sleep stages are reduced compared to lower sleep stages. This results in higher energy expenditure at night, thereby increasing the REE estimated from night recording. Third, the physical activity level on the day before REE measurement might affect the determination of REE at night. While some studies found that the degree of physical activity the day before REE measurement does not affect the REE val- ue itself [25], other experts recommend a 14-hour abstention from vigorous exercise before REE measurement [16]. Fourth, room temperature might affect REE measurement obtained from sleeping subjects. It has been found that a lower ambient temperature results in higher energy expenditure. For example, at a temperature of $16^{\circ} \mathrm{C}$, in comparison to $22^{\circ} \mathrm{C}$, the sleeping metabolic rate increased by $5.7 \%$ [26]. Fifth, also diet-induced energy expenditure could be responsible for higher sleeping REE measurements. Goldberg et al. [27] reported that the overnight metabolic rate increased by $6.7 \%$ in those subjects who were fed a late-night meal. Sixth, seasonal variance might also influence sleeping REE measurement. Plasqui et al. [28] found that the sleeping metabolic rate was significantly decreased by $4.6 \%$ when measured in summer, compared with measurements in winter. In that study, $17 \%$ of intraindividual variation in sleeping metabolic rate was explained by the season. However, the present study found no significant relationship between month of REE measurement and difference between night and morning recordings of REE ( $\mathrm{rs}=-0.2 ; \mathrm{p}=$ 0.273 ). Finally, hormonal differences with respect to circadian variation in body temperature might also influence sleeping energy expenditure in elderly persons. All these factors taken together could be controlled with a standardized protocol for night recording by SWA. The protocol should control for the most likely reasons for overprediction by night recording, i.e. for exercise, eating behavior and room temperature. Prior to night recording, the subjects should have been abstinent from vigorous exercise for $14 \mathrm{~h}$, should not haven taken a late-night meal, and should have a temperature of at least $20^{\circ} \mathrm{C}$ in their sleeping rooms in order to improve SWA measurement of REE. On the other hand, an accurate REE measurement by SWA in the morning also requires some standardization and pretest conditions like those mentioned above, and the users have to be carefully instructed about this.

\section{Accuracy of REE Measurement by SWA}

The SWA significantly overpredicted the mean REE in resting older subjects by $12-14 \%$, compared with indirect calorimetry. Other authors also observed that the SWA overestimates energy expenditure in healthy young adults [4], in middle-aged (44 \pm 12 years) normal subjects [29] and in intensive care patients [7]. By contrast, some authors observed a nonsignificant underestimation of REE (9\%) by SWA in younger subjects [5], and a significant underestimation in obese individuals [6]. 
The agreement between SWA and indirect calorimetry at an individual level was assessed by use of the BlandAltman analysis. Although, in absolute values, the mean difference between the two methods was small (167 $\mathrm{kcal} / 24 \mathrm{~h}$ for morning recording; $187 \mathrm{kcal} / 24 \mathrm{~h}$ for night recording), the agreement may not be suitable for measurement of REE in all individual persons. The individual variations are great, in some subjects ranging from $-8 \%$ to $+32 \%$.

Both morning recording and night recording by SWA overestimated REE in the aged, as compared to indirect calorimetry. The overestimation of REE by SWA in the older subjects is possibly due to age-related changes in skin conductance and thermoregulation, both measured by sensors of the SWA. With increasing age, there is a decrease in blood vessel volume, in the amount of subcutaneous fat mass, and in the number of sweat and sebaceous glands. This results in decreased skin greasing, a deteriorated thermoregulation, and a change in skin conductance. In addition, there are anatomical and physiological changes in the sympathetic nervous system with age [30] which affect sudomotor function [31]. The latency of sympathetic skin response - a transient change in skin voltage (electrodermal activity) - increases significantly with age [10]. This may contribute to a lower conduction. Sympathetic skin response is also affected by hormonal status in the elderly. Estrogen, which decreases with age, significantly inhibits the sympathetic neuron pool at the spinal cord level [32]. All these factors taken together might result in an overestimation of REE. Therefore, we assume that the SWA algorithms developed for estimating REE in younger adults cannot be applied to older ones. The data suggest that the accuracy of SWA measurements seems to be age dependent as they provide a good prediction of energy expenditure in healthy younger and middle-aged adults, but not in children [33] and older adults. We assume that the algorithms were not specifically developed from data on older people. Therefore, the software should be adapted to older adults, as it has recently been done for children [34]. The SWA has the advantage of determining REE by easy handling, and has the potential to be used in epidemiological studies.

The present study for the first time investigated the applicability of the SWA device for determining REE in healthy older adults. Additional studies are needed, examining the SWA monitor for predicting REE in frail older people as well as in underweight and overweight older populations.

\section{Acknowledgment}

We are grateful to the volunteers for participating in this study.

\section{Disclosure Statement}

The authors have nothing to disclose.

\section{References}

-1 Bosy-Westphal A, Eichhorn C, Kutzner D, Illner K, Heller M, Müller MJ: The age-related decline in resting energy expenditure in humans is due to the loss of fat-free mass and to alterations in its metabolically active components. J Nutr 2003;133:2356-2362.

-2 Fredrix EWHM, Soeters PB, Deerenberg IM, Kester ADM, von Meyenfeldt MF, Saris WHM: Resting and sleeping energy expenditure in the elderly. Eur J Clin Nutr 1990;44: 741-747.

3 Roberts SB, Gerard ED: Energy requirements and aging. Public Health Nutr 2005;8: 1028-1036.

4 King GA, Torres N, Potter C, Brooks TJ, Coleman KJ: Comparison of activity monitors to estimate energy cost of treadmill exercise. Med Sci Sports Exerc 2004;36:12441251.
5 Malavolti M, Pietrobelli A, Dugoni M, Poli M, Romagnoli E, de Cristofaro P, Battistini NC: A new device for measuring resting energy expenditure (REE) in healthy subjects. Nutr Metab Cardiovasc Dis 2007; 17:338343.

6 Papazoglou D, Augello G, Tagliaferri M, Savia G, Marzullo P, Maltezos E, Liuzzi A: Evaluation of a multisensor armband in estimating energy expenditure in obese individuals. Obesity (Silver Spring) 2006;14:2217-2223.

$\checkmark 7$ Cereda E, Turrini M, Ciapanna D, Marbello L, Pietrobelli A, Corradi E: Assessing energy expenditure in cancer patients: a pilot validation of a new wearable device. JPEN J Parenter Enteral Nutr 2007;31:502-507.

8 Rokuss K, Kalenka A, Bender HJ, Hinkelbein J: Intensive care patients: determining daily energy expenditure - a comparison of two methods (in German). Anaesthesist 2009;58: 787-794.
-9 Baumgartner RN, Stauber PM, McHugh D, Koehler KM, Garry PJ: Cross-sectional age differences in body composition in persons $60+$ years of age. J Gerontol A Biol Sci Med Sci 1995;50A:M307-M316.

10 Hay JE, Taylor PK, Nukada H: Auditory and inspiratory gasp-evoked sympathetic skin response: age effects. J Neurol Sci 1997;148: 19-23.

11 Khalaj Hedayati K, Bosy-Westphal A, Müller MJ, Dittmar M: Validation of the BIOPAC indirect calorimeter for determining resting energy expenditure in healthy free-living elderly people. Nutr Res 2009;29:531-541.

12 Reeves MM, Davies PS, Bauer J, Battistutta D: Reducing the time period of steady state does not affect the accuracy of energy expenditure measurements by indirect calorimetry. J Appl Physiol 2004;97:130-134. 
13 Weir JB: New methods for calculating metabolic rate with special reference to protein metabolism. J Physiol 1949;109:1-9.

14 Korth O: Körperzusammensetzung und Sympathikotonus als Determinanten des Ruheenergieverbrauchs. Tönning, Der Andere Verlag, 2006.

15 Bader N, Bosy-Westphal A, Dilba B, Müller MJ: Intra- and interindividual variability of resting energy expenditure in healthy male subjects: biological and methodological variability of resting energy expenditure. Brit J Nutr 2005;94:843-849.

16 Compher C, Frankenfield MS, Keim N, Roth-Yousey L, Evidence Analysis Working Group: Best practice methods to apply to measurement of resting metabolic rate in adults: a systematic review. J Am Diet Assoc 2006;106:881-903.

17 Dörhöfer RP, Pirlich M: Das B.I.A. Kompendium. Darmstadt, Data Input, 2007.

-18 Voorrips L, Ravelli ACJ, Dongelmans PCA, Deurenberg P, van Staveren WA: A physical activity questionnaire for the elderly. Med Sci Sports Exerc 1991;23:974-979.

19 Bland JM, Altman DG: Statistical methods for assessing agreement between two methods of clinical measurement. Lancet 1986;i:307-310.
20 Müller MJ, Przyrembel H, Schmidt T: Ernährungsmedizinische Praxis. Methoden Prävention - Behandlung. Berlin, Springer, 2006.

21 Schoffelen PFM, Westerterp KR: Intra-individual variability and adaptation of overnight- and sleeping metabolic rate. Physiol Behav 2008;94:158-163.

22 Katayose Y, Tasaki M, Ogata H, Nakata Y, Tokuyama K, Satoh M: Metabolic rate and fuel utilization during sleep assessed by whole-body indirect calorimetry. Metabolism 2009;58:920-926.

23 Fontvieille AM, Rising R, Spraul M, Larson $\mathrm{DE}$, Ravussin E: Relationship between sleep stages and metabolic rate in humans. Am J Physiol 1994;267:E732-E737.

24 Wouters-Adriaens MP, Westerterp KR: Basal metabolic rate as a proxy for overnight energy expenditure: the effect of age. Br J Nutr 2006;95:1166-1170.

25 Adriaens MP, Schoffelen PF, Westerterp KR Intra-individual variation of basal metabolic rate and the influence of daily habitual physical activity before testing. Br J Nutr 2003;90: 419-423.

26 Westerterp-Plantenga MS, van Marken Lichtenbelt WD, Strobbe H, Schrauwen P: Energy metabolism in humans at a lowered ambient temperature. Eur J Clin Nutr 2002; 56:288-296.

- 27 Goldberg GR, Prentice AM, Davies HL, Murgatroyd PR: Overnight and basal metabolic rates in men and women. Eur J Clin Nutr 1988;42:137-144.
28 Plasqui G, Kester AD, Westerterp KR: Seasonal variation in sleeping metabolic rate, thyroid activity, and leptin. Am J Physiol Endocrinol Metab 2003;285:E338-E343.

29 Bertoli S, Posata A, Battezzati A, Spadafranca A, Testolin G, Bedogni G: Poor agreement between a portable armband and indirect calorimetry in the assessment of resting energy expenditure. Clin Nutr 2008;27:307310.

30 Drory VE, Korczyn AD: Sympathetic skin response: age effect. Neurology 1993;43: $1818-1820$

- 31 Ferrer T, Ramos MJ, Pérez-Sales P, Pérez-Jiménez A, Alvarez E: Sympathetic sudomotor function and aging. Muscle Nerv 1995;18: 395-401.

32 Sahiner T, Aktan E, Kaleli B, Oguzhanoglu A: The effects of postmenopausal hormone replacement therapy on sympathetic skin response. Maturitas 1998;30:85-88.

33 Arvidsson D, Slinde F, Larsson S, Hulthén L: Energy cost of physical activities in children: validation of SenseWear Armband. Med Sci Sports Exerc 2007;39:2076-2084.

34 Calabró MA, Welk GJ, Eisenmann JC: Validation of the SenseWear Pro Armband algorithms in children. Med Sci Sports Exerc 2009;41:1714-1720. 